\title{
Tooth Loss-associated Cognitive Impairment in the Elderly: A Community-based Study in Japan
}

\author{
Hajime Kato ${ }^{1-3}$, Yoshimi Takahashi ${ }^{1}$, Chifumi Iseki ${ }^{1}$, Ryosuke Igari ${ }^{1}$, Hidenori Sato ${ }^{1}$, \\ Hiroyasu Sato ${ }^{1}$, Shingo Koyama ${ }^{1}$, Muneshige Tobita ${ }^{3}$, Toru Kawanami ${ }^{4}$, Mitsuyoshi Iino ${ }^{2}$, \\ Kenichi Ishizawa $^{1}$ and Takeo Kato ${ }^{1,5}$
}

\begin{abstract}
:
Objective Dementia is a major cause of disruption for a healthy life expectancy in Japan. It has been suggested that the number of teeth is a modifiable risk factor for cognitive impairment and dementia. We therefore examined the possible association between the cognitive function and the number of natural and artificial teeth in community-dwelling Japanese elderly individuals.

Methods Among the participants in our prospective, community-based study, 210 elderly individuals (103 men and 107 women; 78.1 \pm 4.9 years; mean age \pm standard deviation) underwent both dental examinations and a Mini-Mental State Examination (MMSE), as well as various medical checkups, in 2016 and 2017.

Results The number of natural teeth was significantly associated with an individual's MMSE score. The percentage of cognitively normal subjects (MMSE scores: 27-30) decreased significantly with a decrease in the number of natural teeth. Among the MMSE items, the calculation ability was significantly and independently associated with the number of natural teeth. Regression was calculated as the predicted score of MMSE $=21+0.3 \times$ (years of schooling $)+0.1 \times$ (number of natural teeth). Among individuals with 19 or fewer natural teeth, those who had a total of 20 teeth or more, including both natural and artificial teeth, had significantly higher MMSE scores than those who had 19 or fewer natural and artificial teeth combined.

Conclusion The number of natural teeth was significantly associated with the cognitive function, especially the calculation ability, and the use of artificial teeth was associated with the preservation of the cognitive function in community-dwelling elderly individuals.
\end{abstract}

Key words: cognitive impairment, dementia, mini-mental state examination, natural teeth, artificial teeth, community-based study

(Intern Med 58: 1411-1416, 2019)

(DOI: 10.2169/internalmedicine.1896-18)

\section{Introduction}

Japan has achieved one of the longest lifespans in the world; in 2016, the average life expectancy was 87.14 years for women and 80.98 years for men (Ministry of Health, Labor, and Welfare 2017). The Japanese government is now trying to extend the "healthy" life expectancy, in which people can live healthy lives without support. The Japanese healthy life expectancy in 2016 was estimated to be 74.79 years for women and 72.14 years for men (Ministry of Health, Labor, and Welfare 2017). Three major causes of disruption of the healthy lives of Japanese people are (1) dementia, (2) cerebrovascular diseases, and (3) diseases of locomotive organs, including bone fractures and arthropathies (Comprehensive Survey of Living Conditions of 2016, Ministry of Health, Labor, and Welfare). To extend the healthy life expectancy, it is therefore important to prevent and/or

${ }^{1}$ Division of Neurology and Clinical Neuroscience, Department of Internal Medicine III, Yamagata University Faculty of Medicine, Japan, ${ }^{2}$ Department of Dentistry, Oral and Maxillofacial-Plastic and Reconstructive Surgery, Yamagata University Faculty of Medicine, Japan, ${ }^{3}$ National Hospital Organization Yonezawa Hospital, Japan, ${ }^{4}$ National Hospital Organization Yamagata Hospital, Japan and ${ }^{5}$ Yamagata City Office, Japan Received: July 29, 2018; Accepted: October 22, 2018; Advance Publication by J-STAGE: January 10, 2019 Correspondence to Dr. Takeo Kato, tkato@med.id.yamagata-u.ac.jp 


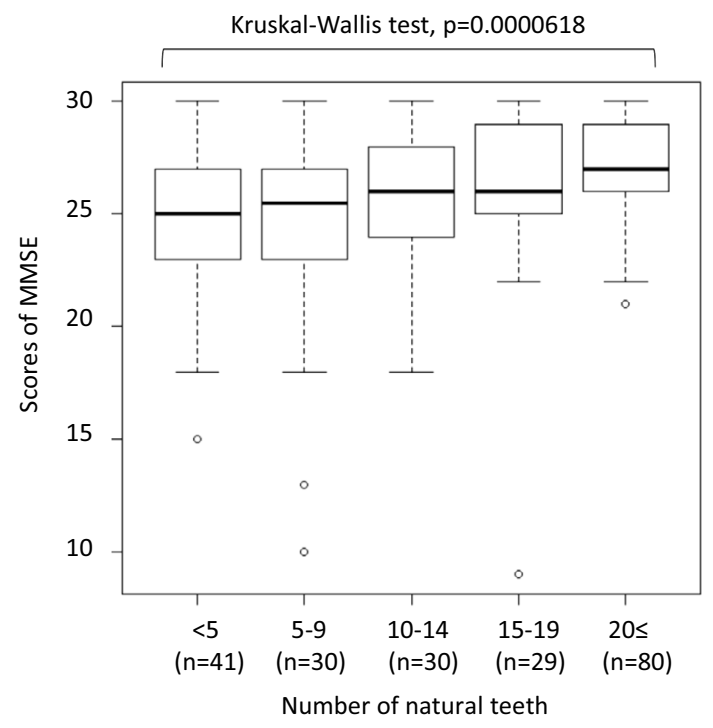

Figure 1. A box-and-whisker plot showing that the number of natural teeth is significantly associated with the MMSE scores (Kruskal-Wallis test, $\mathbf{p}=\mathbf{0 . 0 0 0 0 6 1 8}$ ).

control these diseases.

A variety of risk factors for dementia have been reported, including less education in early life $(<18$ years of age); hypertension, obesity, and hearing loss in midlife (45-65 years of age); and smoking, depression, physical inactivity, social isolation, and diabetes in later life ( $>65$ years of age) (1). Many studies have also reported that tooth loss is associated with cognitive impairment and dementia (2-11). In most cases, tooth loss is the eventual outcome of periodontitis (12-14), making this a potentially preventable/modifiable risk factor for cognitive impairment and dementia.

In the present study, we examined the possible association between the number of teeth and the cognitive function in community-dwelling Japanese elderly individuals. We also examined whether or not the use of artificial teeth could preserve the cognitive function in these individuals.

\section{Materials and Methods}

\section{Subjects}

In 2000, we began a prospective, community-based investigation of elderly residents in Takahata, a town in a rural area of Yamagata Prefecture, Japan $(15,16)$. Participants have been followed up until now. Subjects in the present study were 210 participants [103 men and 107 women; 78.1 \pm 4.9 years of age; mean \pm standard deviation (SD)] who had undergone both dental examinations and a Mini-Mental State Examination (MMSE), in addition to various medical checkups, in 2016 and 2017.

The study was approved by the Medical Ethics Committee of the Yamagata University Faculty of Medicine. All participants gave their written informed consent.

\section{The dental examination}

One dentist (HK) carefully examined the dental status of each participant, including the numbers of natural and artificial teeth. "Natural teeth" were defined as an individual's own teeth with roots, including healthy, carious, or treated teeth (such as resined, crowned, inlay, and abutment tooth for bridge). Wisdom teeth (third molars) were also counted but not included in the number of natural teeth, as they were observed in only roughly $10 \%$ of the subjects and were mostly nonfunctional. "Artificial teeth" were defined as prosthetic replacements for missing teeth, such as dentures, pontics for bridges, or implants. The dentist had no information on the results of MMSEs and medical checkups.

\section{The MMSE}

The cognitive function of each subject was evaluated by the MMSE, which is a measure of the cognitive function used worldwide. Scores range from 0 to 30, with the best being 30. The MMSE contains 11 questions in 8 categories, including orientation to time, orientation to place, registration, calculation, recall, language, repetition, and responses to four complex commands (17). Subjects with an MMSE score of $\geq 27$ were regarded as cognitively normal. Subjects with an MMSE score of 22-26 were suspected of having mild cognitive impairment (MCI), and those with a score of $\leq 21$ were suspected of having dementia.

\section{Clinical and laboratory examinations}

The number of years of schooling was obtained at a faceto-face medical interview. The health check included a blood pressure measurement, neurological examination, and blood tests (including $\mathrm{HbA} 1 \mathrm{c}$ ). The estimated glomerular filtration rate (eGFR) was calculated from the serum creatinine value and age using the abbreviated Modification of Diet in Renal Disease equation modified by the Japanese coefficient (18).

\section{Statistical analyses}

Student's $t$-test, the Mann-Whitney U test, Kruskal-Wallis test, Cochran-Armitage trend test, Spearman's rank correlation test, and a multiple regression analysis were used for statistical analyses. The Results section describes which statistical tests were used for each comparison. A probability value of $\mathrm{p}<0.05$ was considered statistically significant. All analyses were performed using the R package 3.2.0 (http://w ww.r-project.org/) and EZR (19).

\section{Results}

The mean MMSE score in the subjects examined was 25.9 \pm 3.4 (mean $\pm S D$; range 9-30). First, we examined the possible association between the number of natural teeth and the cognitive function. The results showed that the number of natural teeth was significantly associated with the MMSE score (Kruskal-Wallis test, $\mathrm{p}=0.0000618$ ) (Fig. 1). Aging is known to be a risk factor for both cognitive impairment and 


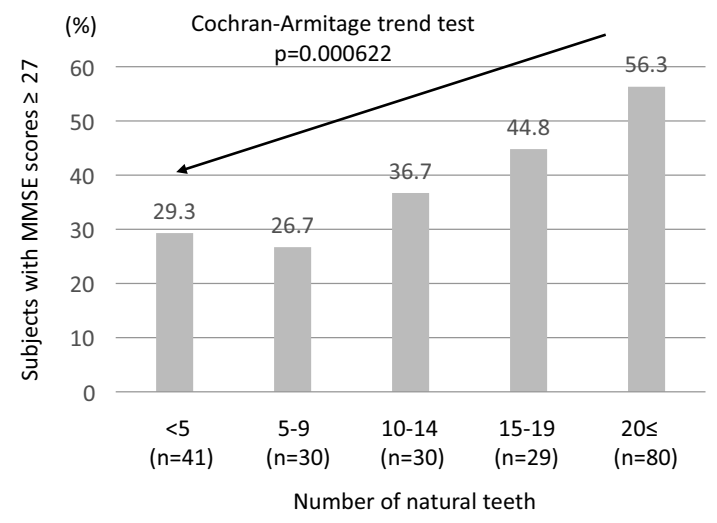

Figure 2. The percentage of cognitively normal subjects (MMSE scores of 27-30) decreases with a decrease in the number of natural teeth (Cochran-Armitage trend test, $p=0.000622$ ).

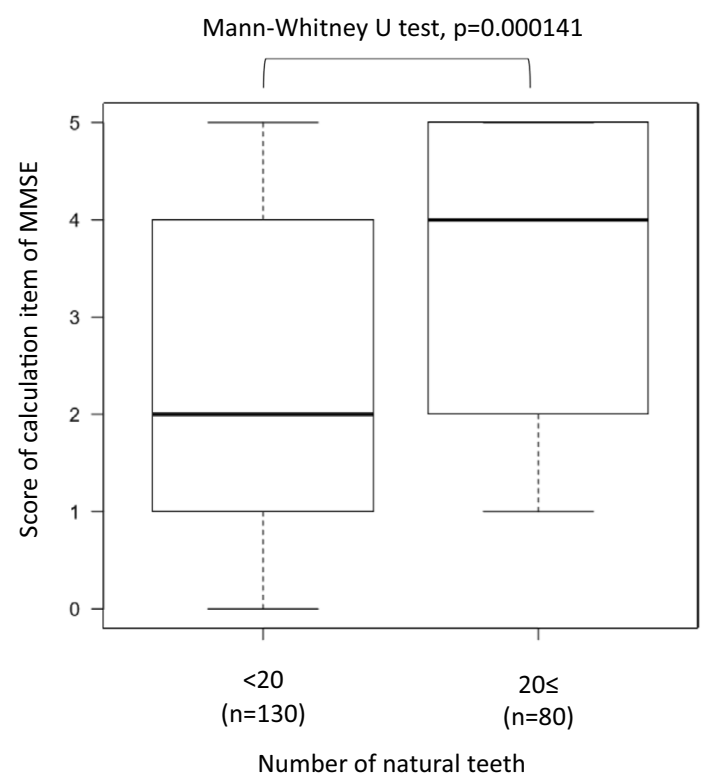

Figure 3. A box-and-whisker plot showing that the subjects with $\geq 20$ natural teeth had significantly higher scores for the calculation item of the MMSE than those with $\leq 19$ natural teeth (Mann-Whitney $U$ test, $p=0.000141$ ). The median scores were 4 and 2 in the subjects with $\geq 20$ and $\leq 19$ natural teeth, respectively. The scores for the calculation item of the MMSE ranged from 0 to 5 in each subject.

tooth loss; therefore, we performed a multiple regression analysis in order to adjust the effect of age on the findings, resulting in a significant correlation between the number of natural teeth and the scores of MMSE, independent of age (Estimate $=0.1 ; 95 \%$ confidence of interval $=0.048-0.15 ; \mathrm{p}=$ $0.00023)$. The percentage of cognitively normal subjects (MMSE scores: 27-30) decreased with the decrease in the number of natural teeth (Cochran-Armitage trend test, $\mathrm{p}=$ 0.000622) (Fig. 2). Among the 11 items of the MMSE, the calculation ability was significantly and independently associated with the number of natural teeth (multiple regression analysis, Estimate $=0.923$; Standard Error $=0.398$; $\mathrm{t}$ value $=$ $2.32 ; \mathrm{p}=0.0216)$. The scores for the calculation item on the
Table 1. Spearman's Correlation with MMSE Score.

\begin{tabular}{lcc}
\hline & mean $\pm \mathrm{SD}$ (range) & $\mathrm{p}$ value \\
\hline Age (years) & $78.1 \pm 4.9(74-87)$ & 0.0031 \\
Gender (Female/Male) & $107 / 103$ & $0.64 *$ \\
Years of education & $10.7 \pm 2.0(6-18)$ & 0.00065 \\
Number of natural teeth & $14.7 \pm 9.3(0-28)$ & $1.4 \times 10^{-6}$ \\
Dental brushing ( /day) & $1.8 \pm 0.9(0-5)$ & 0.063 \\
BMI $\left(\mathrm{kg} / \mathrm{m}^{2}\right)$ & $23.0 \pm 3.9(14.1-40.1)$ & 0.96 \\
Systolic BP $(\mathrm{mmHg})$ & $139.0 \pm 17.0(90-188)$ & 0.19 \\
Diastolic BP $(\mathrm{mmHg})$ & $74.9 \pm 10.0(44-106)$ & 0.27 \\
Fasting blood sugar $(\mathrm{mg} / \mathrm{dL})$ & $107.4 \pm 23.6(78-263)$ & 0.85 \\
HbA1c $(\%)$ & $6.0 \pm 0.7(4.8-11.4)$ & 0.55 \\
Trigliceride $(\mathrm{mg} / \mathrm{dL})$ & $108.8 \pm 61.3(31-567)$ & 0.38 \\
LDLc $(\mathrm{mg} / \mathrm{dL})$ & $113.1 \pm 30.5(44-234)$ & 0.38 \\
HDLc $(\mathrm{mg} / \mathrm{dL})$ & $60.8 \pm 17.2(27-182)$ & 0.87 \\
AST $(\mathrm{U} / \mathrm{L})$ & $20.7 \pm 13.9(11-150)$ & 0.16 \\
ALT $(\mathrm{U} / \mathrm{L})$ & $20.1 \pm 13.7(7-175)$ & 0.54 \\
$\gamma$-GTP $(\mathrm{U} / \mathrm{L})$ & $35.0 \pm 50.6(9-406)$ & 0.66 \\
Uric acid $(\mathrm{mg} / \mathrm{dL})$ & $5.3 \pm 1.3(1.7-8.8)$ & 0.17 \\
Creatinine $(\mathrm{mg} / \mathrm{dL})$ & $0.8 \pm 0.2(0.4-1.5)$ & 0.59 \\
eGFR $\left(\mathrm{mL} / \mathrm{min} / 1.73 \mathrm{~m}^{2}\right)$ & $64.2 \pm 14.5(26.6-117.4)$ & 0.78 \\
WBC $\left(\times 10^{2} / \mu \mathrm{L}\right)$ & $53.2 \pm 14.1(26-98)$ & 0.66 \\
Hb $(\mathrm{g} / \mathrm{dL})$ & $13.4 \pm 1.3(10.1-17.1)$ & 0.56 \\
PLT $\left(\times 10^{4} / \mu \mathrm{L}\right)$ & $21.8 \pm 6.1(1.5-39.7)$ & 0.15 \\
\hline
\end{tabular}

*Mann-Whitney U test.

MMSE: mini-mental state examination

MMSE ranged from 0 to 5 in each subject. Fig. 3 shows that the subjects with $\geq 20$ natural teeth had significantly higher scores for the calculation item than those with $\leq 19$ natural teeth (Mann-Whitney U test, $\mathrm{p}=0.000141$ ). The median scores for the calculation item were 4 and 2 in the subjects with $\geq 20$ and $\leq 19$ natural teeth, respectively (Fig. 3). None of the other items were significantly associated with the number of natural teeth (data not shown).

To elucidate the demographic/laboratory findings related to the MMSE score, Spearman's rank correlation test was performed and showed that the age, education level (years of schooling), and number of natural teeth were significantly related to the MMSE score (Table 1). As shown in Table 2, the MMSE score was predicted based on the length of education (years of schooling) and the number of natural teeth, independent of age. The predicted MMSE score was equal to $21+0.3 \times$ (years of schooling $)+0.1 \times$ (number of natural teeth) (Table 2). Both the length of education (years of schooling) and the number of natural teeth were significant and independent predictors of the MMSE score (Table 2).

We next examined whether or not the use of artificial teeth could influence the cognitive function. To clarify this point, we examined a subgroup of participants who had $\leq 19$ natural teeth $(\mathrm{n}=130)$. These subjects were further divided into two groups: those with a total of $\geq 20$ teeth $(n=111)$ and those with a total of $\leq 19$ teeth $(n=19)$, including both natural and artificial teeth (Fig. 4). There was no significant difference in the number of natural teeth between the two groups $(8.4 \pm 6.5$ vs. $10.1 \pm 5.5$, mean $\pm \mathrm{SD}$; Student's $t$-test, $\mathrm{p}=$ 
Table 2. Multiple Regression Analysis for Estimation of MMSE Score.

\begin{tabular}{lcccccc}
\hline & Estimates & $95 \% \mathrm{CI}$ & $\mathrm{p}$ value & Estimates & $95 \% \mathrm{CI}$ & $\mathrm{p}$ value \\
\hline (Intercept) & 23.3 & $15.1-31.4$ & $6.57 \times 10^{-8}$ & 21.1 & $18.9-23.2$ & $1.5 \times 10^{-45}$ \\
Age & -0.03 & $-0.12-0.07$ & 0.58 & & & \\
Education (years) & 0.32 & $0.12-0.52$ & 0.0017 & 0.33 & $0.13-0.53$ & 0.0011 \\
Natural tooth number & 0.10 & $0.05-0.14$ & $6.4 \times 10^{-5}$ & 0.10 & $0.06-0.15$ & $3.0 \times 10^{-6}$ \\
\hline
\end{tabular}

Predicted MMSE score $=21+0.3 \times($ years of schooling $)+0.1 \times$ (natural tooth number $)$.

CI: confidence of interval

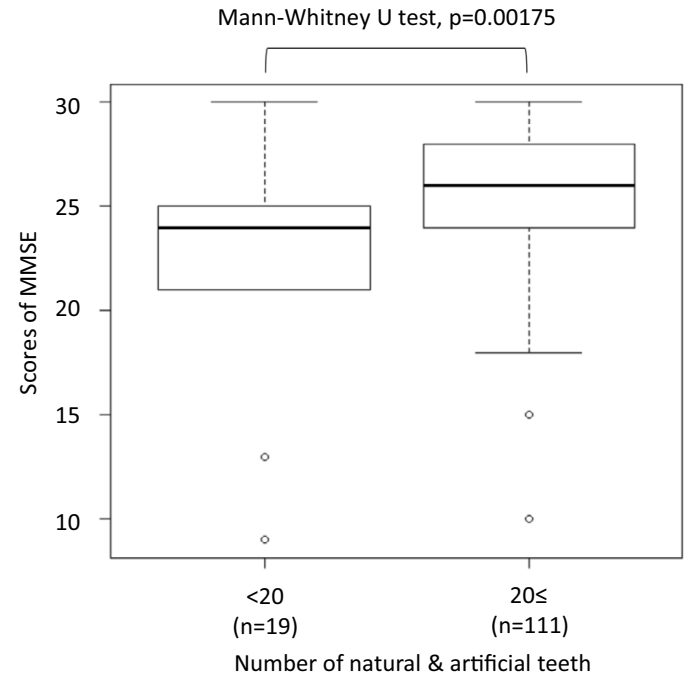

\begin{tabular}{|c|c|c|c|}
\hline Natural \& artificial teeth & $<20$ & $20 \leq$ & Student $t$ test \\
\hline Natural teeth & $10.1 \pm 5.5^{*}$ & $8.4 \pm 6.5^{*}$ & $\mathrm{p}=0.3$ \\
\hline Artificial teeth & $2.0 \pm 3.6^{*}$ & $18.5 \pm 7.2^{*}$ & $\mathrm{p}=2.53 \times 10^{-17}$ \\
\hline \multicolumn{4}{|c}{$*$ mean \pm SD }
\end{tabular}

Figure 4. Among the participants who had $\leq 19$ natural teeth ( $n=130$ ), those with a total of $\geq 20$ teeth, including both natural and artificial teeth, had significantly higher MMSE scores than those with $\leq 19$ total teeth (Mann-Whitney $U$ test, $p=0.00175$ ).

0.3) (Fig. 4), whereas a remarkable difference between them was found in the number of artificial teeth (18.5 \pm 7.2 vs. 2.0 \pm 3.6 , mean \pm SD; Student's $t$-test, $\mathrm{p}=2.53 \times 10^{-17}$ ) (Fig. 4). Subjects who had a total of $\geq 20$ teeth, including both natural and artificial teeth, had significantly higher MMSE scores than those who had a total of $\leq 19$ teeth (Mann-Whitney U test, $\mathrm{p}=0.00175$ ) (Fig. 4).

\section{Discussion}

The present study showed that the number of natural teeth was significantly associated with the MMSE scores in community-dwelling Japanese elderly individuals, independent of age, with the MMSE score estimated as 21+0.3x (years of schooling) $+0.1 \times($ number of natural teeth). Among the items of the MMSE, the calculation ability was significantly associated with the number of natural teeth. In addition, the use of artificial teeth was found to be associated with the preservation of the cognitive function in these subjects.

Previous studies have shown a significant association be- tween the number of teeth and the cognitive function/dementia (2-11). In this respect, the present study confirmed the findings of these previous reports. In addition, our study revealed that, among the MMSE items, the calculation ability was significantly associated with the number of natural teeth; no previous studies have examined the association between each item of the MMSE and the number of natural teeth.

The mechanism by which cognitive impairment was associated with tooth loss remains unknown; however, there are some possibilities. One is the presence of periodontitis for a long time before tooth loss. In most cases, tooth loss is considered to be the final result of periodontitis (12-14). The inflammatory process associated with periodontitis may induce various cytokines and immunological responses that may adversely affect systemic organs and tissues, including the brain (20-22). It was shown that periodontitis exacerbated the brain inflammation triggered by brain amyloid- $\beta$ deposition in a mouse model of Alzheimer's disease (23). Oral bacteria have also been neuropathologically found even in the brain of patients with Alzheimer's disease $(24,25)$. Another possibility is poor nutrition caused by periodontitis and/or tooth loss (26-28). Low B vitamins and high homocysteine concentrations were shown to predict cognitive decline in aging men (26). The third possibility is a reduced ability to masticate due to tooth loss $(29,30)$. In animal models, long-term masticatory dysfunction has been shown to induce memory impairment and neuronal cell loss in the hippocampus (31). It has also been reported that mastication may be associated with the hippocampal function via the expression of a neurotrophic factor in mice (32). In the present study, however, tooth loss was significantly associated with the calculation ability, but not with memory impairment, among the items of the MMSE. The calculation ability in the brain is localized to the dominant parietal lobe $(33,34)$, not the hippocampus. Memory loss and neuronal cell loss in the hippocampus are the cardinal features of Alzheimer's disease (35). Therefore, it is possible that tooth lossassociated cognitive impairment (TAC) may be derived from a pathological process different from Alzheimer's disease. The pathophysiological mechanism by which calculation is impaired in TAC therefore remains to be elucidated.

The present study revealed that the use of artificial teeth was associated with the preservation of the cognitive function in community-dwelling Japanese elderly individuals. However, their causal relationship remains unclear, as the 
present study was a cross-sectional study. Therefore, we cannot rule out the possibility that cognitively-impaired elderly individuals may not pay attention to their oral hygiene, resulting in their refusal of dental therapy. In a six-year longitudinal study examining institutionalized non-demented elderly individuals in nursing homes or homes for the aged, Shimazaki et al. observed that the incidence of mental impairment was greater in the subjects with few or no teeth not using dentures than in those using dentures; however, it was not significant after multivariate adjustment (36). In their study, the dentition status was examined by two trained dentists; however, the mental health status was obtained from nurses belonging to each home (36). They did not use the MMSE, the most widely used screening tool for providing an overall measure of cognitive impairment. It has also been reported that elderly individuals with few teeth and no dentures but not those with dentures developed dementia more frequently than those with $\geq 20$ teeth during a followup period of 4 years (37). In that study, the tooth number was self-reported by participants, and the records of dementia onset were obtained from municipalities in charge of the public long-term care insurance system (37). In the present study, the tooth number was closely evaluated by a dentist, and the cognitive state was determined using the MMSE; therefore, the present study identified subjects with cognitive impairment before the onset of dementia. Despite the difference in the methods used among the three studies, it seems that the use of artificial teeth or dentures may aid in preserving the cognitive function in elderly individuals.

The limitation of the present study is that it is a crosssectional study. For example, we demonstrated a significant association between tooth loss and cognitive impairment in community-dwelling elderly individuals; however, we were unable to determine whether tooth loss caused cognitive impairment or cognitive impairment caused tooth loss. In a three-year prospective cohort study of cognitively normal subjects, Okamoto et al. found that subjects with fewer teeth developed mild memory impairment more frequently than those with more teeth (2). Dintica et al. followed dementiafree participants for nine years and found that tooth loss was associated with a steeper cognitive decline during the follow-up period than tooth retention (9). Li et al. also observed that those who had fewer teeth tended to show a more rapid cognitive decline than those with more teeth in their 13-year longitudinal study (7). Taken together, these findings suggest that tooth loss may be a cause of cognitive impairment. Cognitive impairment and dementia are serious problems in aging societies, including Japan. The preservation of teeth by maintaining the dental health and/or using artificial teeth may be one possible measure for helping to preserve the cognitive function in the elderly.

In conclusion, tooth loss may be a cause of cognitive impairment, and the use of artificial teeth may help preserve the cognitive function in the elderly. Tooth loss-associated cognitive impairment may be characterized by an impaired calculation ability. Further longitudinal studies on humans and experimental animals are needed to clarify the causal relationship between the oral health status and the cognitive function.

The authors state that they have no Conflict of Interest (COI).

\section{Acknowledgement}

The authors thank the staff of Takahata Town Office for cooperating with us to perform the present study.

\section{References}

1. Livingston G, Sommerlad A, Orgeta V, et al. Dementia prevention, intervention, and care. Lancet 390: 2673-2734, 2017.

2. Okamoto N, Morikawa M, Tomioka K, et al. Association between tooth loss and the development of mild memory impairment in the elderly: the Fujiwara-kyo study. J Alzheimers Dis 44: 777-786, 2015.

3. Tsakos G, Watt RG, Rouxel PL, de Oliveira C, Demakakos P. Tooth loss associated with physical and cognitive decline in older adults. J Am Geriatr Soc 63: 91-99, 2015.

4. Luo J, Wu B, Zhao Q, et al. Association between tooth loss and cognitive function among 3063 Chinese older adults: a community-based study. PLoS One 10: e0120986, 2015.

5. Takeuchi K, Izumi M, Furuta M, et al. Posterior teeth occlusion associated with cognitive function in nursing home older residents: a cross-sectional observational study. PLoS One 10: e0141737, 2015.

6. Zhu J, Li X, Zhu F, et al. Multiple tooth loss is associated with vascular cognitive impairment in subjects with acute ischemic stroke. J Periodontal Res 50: 683-688, 2015.

7. Li J, Xu H, Pan W, Wu B. Association between tooth loss and cognitive decline: a 13-year longitudinal study of Chinese older adults. PLoS One 12: e0171404, 2017.

8. Tonsekar PP, Jiang SS, Yue G. Periodontal disease, tooth loss and dementia: is there a link? A systematic review. Gerodontology 34: 151-163, 2017.

9. Dintica CS, Rizzuto D, Marseglia A, et al. Tooth loss is associated with accelerated cognitive decline and volumetric brain differences: a population-based study. Neurobiol Aging 67: 23-30, 2018.

10. Takeuchi K, Ohara T, Furuta M, et al. Tooth loss and risk of dementia in the community: the Hisayama study. J Am Geriatr Soc 65: e95-e100, 2017.

11. Oh B, Han D-H, Han K-T, et al. Association between residual teeth number in later life and incidence of dementia: a systematic review and meta-analysis. BMC Geriatrics 18: 48-56, 2018.

12. Al-Shammari KF, Al-Khabbaz AK, Al-Ansari JM, Neiva R, Wang HL. Risk indicators for tooth loss due to periodontal disease. J Periodontol 76: 1910-1918, 2005.

13. Martinez-Canut P. Predictors of tooth loss due to periodontal disease in patients following long-term periodontal maintenance. J Clin Periodontol 42: 1115-1125, 2015.

14. Chambrone L, Chambrone D, Lima LA, Chambrone LA. Predictors of tooth loss during long-term periodontal maintenance: a systematic review of observational studies. J Clin Periodontol 37: 675-684, 2010.

15. Kato T, Sato H, Emi M, et al. Segmental copy number loss of SFMBT1 gene in elderly individuals with ventriculomegaly: a community-based study. Intern Med 50: 297-303, 2011.

16. Takahashi $Y$, Iseki $C$, Wada $M$, et al. Impaired glucose metabolism slows executive function independent of cerebral ischemic lesions in Japanese elderly: the Takahata study. Intern Med 50: 16711678, 2011.

17. Tuijl JP, Scholte EM, de Craen AJM, van der Mast RC. Screening for cognitive impairment in older general hospital patients: com- 
parison of the six-item cognitive test with the Mini-Mental Status Examination. Int J Geriatr Psychiatry 27: 755-762, 2012.

18. Imai E, Horio M, Nitta $K$, et al. Modification of the modification of diet in renal disease (MDRD) study equation for Japan. Am J Kidney Dis 50: 927-937, 2007.

19. Kanda Y. Investigation of the freely available easy-to-use software 'EZR' for medical statistics. Bone Marrow Transplant 48: 452458, 2013.

20. Kamer AR, Craig RG, Dasanayake AP, Brys M, Glodzik-Sobanska L, de Leon MJ. Inflammation and Alzheimer's disease: possible role of periodontal diseases. Alzheimer's Dement 4: 242-250, 2008.

21. Watts A, Crimmins EM, Gatz M. Inflammation as a potential mediator for the association between periodontal disease and Alzheimer's disease. Neuropsychiatr Dis Treat 4: 865-876, 2008.

22. Bretz WA, Weyant RJ, Corby PM, et al. Systemic inflammatory markers, periodontal diseases, and periodontal infections in an elderly population. J Am Geriatr Soc 53: 1532-1537, 2005.

23. Ishida $\mathrm{N}$, Ishihara $\mathrm{Y}$, Ishida $\mathrm{K}$, et al. Periodontitis induced by bacterial infection exacerbates features of Alzheimer's disease in transgenic mice. NPJ Aging Mech Dis 3: 15, 2017.

24. Riviere GR, Riviere KH, Smith KS. Molecular and immunological evidence of oral Treponema in the human brain and their association with Alzheimer's disease. Oral Microbiol Immunol 17: 113118, 2002.

25. Ranjan R, Abhinay A, Mishra M. Can oral microbial infections be a risk factor for neurodegeneration? A review of the literature. Neurol India 66: 344-351, 2018.

26. Tucker KL, Qiao N, Scott T, Rosenberg I, Spiro A 3rd. High homocysteine and low B vitamins predict cognitive decline in aging men: the Veterans Affairs Normative Aging Study. Am J Clin Nutr 82: 627-635, 2005.

27. Krall E, Hayes C, Garcia R. How dentition status and masticatory function affect nutrient intake. J Am Dent Assoc 129: 1261-1269, 1998.

28. Kim JM, Stewart R, Prince M, Kim SW, Yang SJ, Shin IS, Yoon
JS. Dental health, nutritional status and recent-onset dementia in a Korean community population. Int J Geriatr Psychiatry 22: 850855, 2007.

29. De Cicco V, Barresi M, Tramonti Fantozzi MP, Cataldo E, Parisi V, Manzoni D. Oral implant-prostheses: new teeth for a brighter brain. PLoS One 11: e0148715, 2016.

30. Ono Y, Yamamoto T, Kubo KY, Onozuka M. Occlusion and brain function: mastication as a prevention of cognitive dysfunction. J Oral Rehabil 37: 624-640, 2010.

31. Oue H, Miyamoto Y, Okada S, et al. Tooth loss induces memory impairment and neuronal cell loss in APP transgenic mice. Behav Brain Res 252: 318-325, 2013.

32. Fukushima-Nakayama Y, Ono T, Hayashi M, et al. Reduced mastication impairs memory function. J Dent Res 96: 1058-1066, 2017.

33. Whalen J, McCloskey M, Ronald P, Lesser RP, Gordon B. Localizing arithmetic processes in the brain: evidence from a transient deficit during cortical stimulation. J Cogn Neurosci 9: 409-417, 1997.

34. Roux F-E, Boukhatem L, Draper L. Cortical calculation localization using electrostimulation. J Neurosurg 110: 1291-1299, 2009.

35. Moodley KK, Chan D. The hippocampus in neurodegenerative disease. Front Neurol Neurosci 34: 95-108, 2014.

36. Shimazaki Y, Soh I, Saito T, et al. Influence of dentition status on physical disability, mental impairment and mortality in institutionalized elderly people. J Dent Res 80: 340-345, 2001.

37. Yamamoto T, Kondo K, Hirai H, Nakade M, Aida J, Hirata Y. Association between self-reported dental health status and onset of dementia: a 4-year prospective cohort study of older Japanese adults from the Aichi Gerontological Evaluation Study (AGES) Project. Psychosom Med 74: 241-248, 2012.

The Internal Medicine is an Open Access journal distributed under the Creative Commons Attribution-NonCommercial-NoDerivatives 4.0 International License. To view the details of this license, please visit (https://creativecommons.org/licenses/ by-nc-nd/4.0/).

(C) 2019 The Japanese Society of Internal Medicine Intern Med 58: 1411-1416, 2019 